\title{
Erratum: Orientation dynamics of asymmetric rotors using random phase wave functions [Phys. Rev. A 91, 063420 (2015)]
}

\author{
Shimshon Kallush and Sharly Fleischer \\ (Received 12 September 2015; published 8 October 2015)
}

DOI: 10.1103/PhysRevA.92.049901

PACS number(s): $37.10 . \mathrm{Vz}, 31.15 . \mathrm{xv}, 42.50 . \mathrm{Md}, 82.53 . \mathrm{Kp}, 99.10 . \mathrm{Cd}$

Following publication we have found that the assignment of moments of inertia to the principal axes of the $\mathrm{SO}_{2}$ molecule was incorrect in our original paper [Fig. 1(d)].

This misassignment of axes resulted in the terahertz- (THz-) induced time-dependent orientation presented throughout our original paper. We corrected the assignment of axes, recalculated the orientation dynamics and present here a correct version of Fig. 3(c) as a representative figure of the THz-induced orientation dynamics of $\mathrm{SO}_{2}$.

The $\mathrm{SO}_{2}$ molecule served merely as an example for the Random Phase Wave Function (RPWF) method in the original paper. Since all of the RPWF calculations were compared with the exact method calculations with the same misassignment of principal axes, all of our original conclusions and statements regarding the RPWF method, its efficiency, and convergence dependence on the initial temperature and THZ field amplitude remain valid as well as its promising application to rotational dynamics of asymmetric molecules.

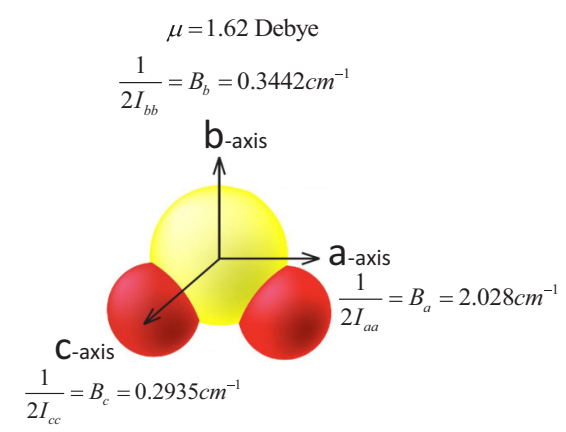

FIG. 1. (Color online) (d) Sketch of the $\mathrm{SO}_{2}$ molecule with the correct assignment of axes.

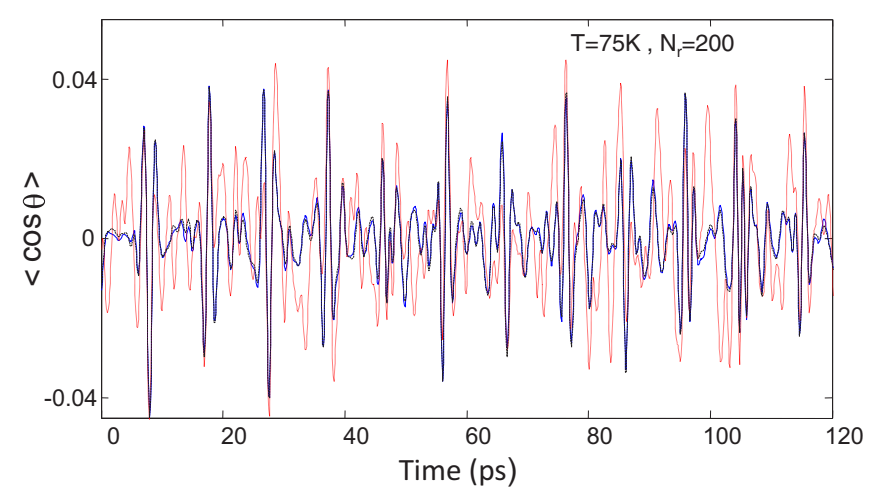

FIG. 3. (Color online) (c) Representative dynamics of $\mathrm{SO}_{2}$ calculated by exact dynamics simulation (blue curve), the RPWF calculation (dashed black curve) with 200 realizations, and the RPWF calculation with a single realization (dotted red curve). 